\title{
MAPGN: MASKED POINTER-GENERATOR NETWORK FOR SEQUENCE-TO-SEQUENCE PRE-TRAINING
}

\author{
Mana Ihori, Naoki Makishima, Tomohiro Tanaka, Akihiko Takashima, Shota Orihashi, Ryo Masumura
}

\author{
NTT Media Intelligence Laboratories, NTT Corporation, Japan
}

\begin{abstract}
This paper presents a self-supervised learning method for pointergenerator networks to improve spoken-text normalization. Spokentext normalization that converts spoken-style text into style normalized text is becoming an important technology for improving subsequent processing such as machine translation and summarization. The most successful spoken-text normalization method to date is sequence-to-sequence (seq2seq) mapping using pointer-generator networks that possess a copy mechanism from an input sequence. However, these models require a large amount of paired data of spoken-style text and style normalized text, and it is difficult to prepare such a volume of data. In order to construct spoken-text normalization model from the limited paired data, we focus on self-supervised learning which can utilize unpaired text data to improve seq2seq models. Unfortunately, conventional self-supervised learning methods do not assume that pointer-generator networks are utilized. Therefore, we propose a novel self-supervised learning method, MAsked Pointer-Generator Network (MAPGN). The proposed method can effectively pre-train the pointer-generator network by learning to fill masked tokens using the copy mechanism. Our experiments demonstrate that MAPGN is more effective for pointer-generator networks than the conventional self-supervised learning methods in two spoken-text normalization tasks.
\end{abstract}

Index Terms - sequence-to-sequence pre-training, pointergenerator networks, self-supervised learning, spoken-text normalization

\section{INTRODUCTION}

With the rise of various automatic speech recognition (ASR) applications such as smart speakers [1, 2] and automatic dictation systems [3 4], it has become increasingly important to accurately process spoken-style text, i.e., the transcribed text from spoken utterances. Spoken-style text often includes disfluencies such as redundant expressions, and various minority spoken expressions (e.g., dialects) because ASR systems convert speech into text in a literal manner. Spoken-style text adversely affects subsequent natural language processing (e.g., machine translation, summarization) because these technologies are often developed to handle written-style text which is text with majority expressions, and no disfluencies or redundant expressions. Thus, it is required to convert spoken-style text (including disfluencies and dialects) into style normalized text (which excludes disfluencies and dialects). In this paper, we aim to improve spoken-text normalization.

Spoken-text normalization tasks are considered as monolingual translation [5] that is regarded as sequence-to-sequence (seq2seq) mapping from text to text. In recent studies, fully neural networkbased seq2seq models [6] have shown effective performance in various monolingual translation tasks such as summarization [7 8], paraphrase generation [9,10] and disfluency detection [11 12]. In particular, seq2seq models based on pointer-generator networks [13] have been utilized recently [14 15]. Pointer-generator networks are effective for monolingual translation tasks because they contain a copy mechanism that copies tokens from a source text to help generate infrequent tokens. Pointer-generator networks have reportedly outperformed attention-based encoder-decoder networks in spoken-text normalization task [16].

To construct seq2seq models for spoken-text normalization, a large amount of paired data of spoken-style text and style normalized text are necessary. However, to make these paired data, we need to prepare manual transcriptions of spoken utterances, and the text-style of these transcriptions needs to be normalized manually. Thus, it is costly and time-consuming to make a large amount of paired data. To mitigate this problem, we use self-supervised learning which has been gaining a lot of attention in recent years. Selfsupervised learning is one form of unsupervised learning where unpaired data is only employed for designing supervised training settings. In natural language processing, self-supervised learning has been improving in natural language generation and natural language understanding [17]-19]. Unfortunately, conventional self-supervised learning methods for seq2seq models do not assume that pointergenerator networks are utilized [20-22]. In practice, the conventional methods are insufficient for pointer-generator networks because they cannot learn to copy tokens from a source text explicitly.

In this paper, we propose a novel self-supervised learning method for pointer-generator networks. The proposed method, MAsked Pointer-Generator Network (MAPGN), is an extension of MAsked Sequence-to-Sequence pre-training (MASS) [20]. MASS pre-trains a seq2seq model by predicting the masked tokens taking the masked sequence as input. In contract, MAPGN can pre-train a copy mechanism efficiently by learning to choose whether to copy or generate tokens with masked tokens. Our experiments demonstrate that the proposed method is effective for pointer-generator networks with less paired data in two spoken-text normalization tasks, dialect conversion task and spoken-to-written-style conversion task.

\section{POINTER-GENERATOR NETWORKS}

This section defines spoken-style normalization with pointer-generator networks. We define spoken-style text as $\boldsymbol{X}=\left\{x_{1}, \cdots, x_{M}\right\}$ and style normalized text as $\boldsymbol{Y}=\left\{y_{1}, \cdots, y_{N}\right\}$, where $x_{m}$ and $y_{n}$ are tokens in the spoken-style and style normalized text, respectively.

The pointer-generator network predicts generation probabilities of a written-style text $\boldsymbol{Y}$ given a spoken-style text $\boldsymbol{X}$. The generation probability of $\boldsymbol{Y}$ is defined as

$$
P(\boldsymbol{Y} \mid \boldsymbol{X} ; \boldsymbol{\Theta})=\prod_{n=1}^{N} P\left(y_{n} \mid y_{1: n-1}, \boldsymbol{X} ; \boldsymbol{\Theta}\right),
$$

where $\Theta=\left\{\theta_{\text {enc }}, \theta_{\text {dec }}\right\}$ represents model parameter sets. $\theta_{\text {enc }}$ and $\theta_{\text {dec }}$ are trainable parameter sets with an encoder and a decoder, respectively. $P\left(y_{n} \mid y_{1: n-1}, \boldsymbol{X} ; \boldsymbol{\Theta}\right)$ can be computed with the encoder 


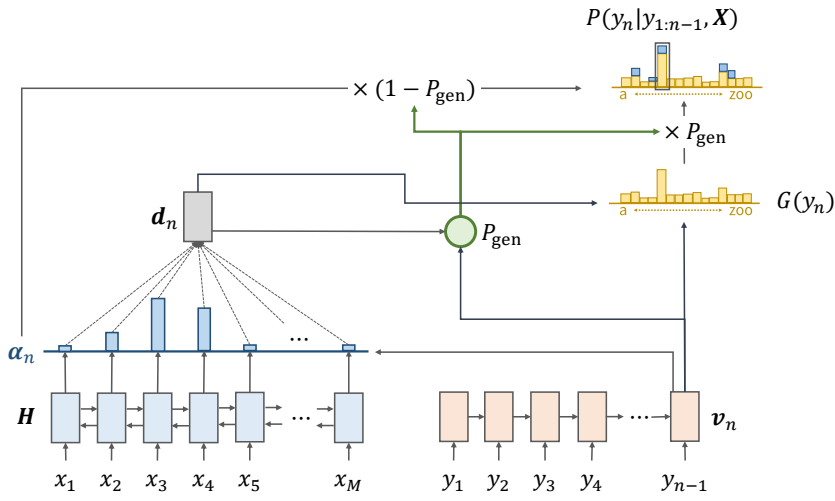

Fig. 1: The network structure of a pointer-generator network.

and the decoder with a copy mechanism. Fig. 1 shows the network structure of the pointer-generator network.

The encoder converts an input sequence $\boldsymbol{X}$ into the hidden representations $\boldsymbol{H}=\left\{\boldsymbol{h}_{1}, \cdots, \boldsymbol{h}_{M}\right\}$. These hidden representations are produced by an arbitrary network such as bidirectional recurrent neural networks (RNNs) [23] or a Transformer encoder [24]. The decoder computes both copying tokens via pointing and generating tokens from a fixed vocabulary based on a copy mechanism to compute the generation probabilities. First, the decoder converts tokens from the first token to the $n-1$-th token into hidden vector $\boldsymbol{v}_{n}$. The hidden vector is produced by an arbitrary network such as unidirectional RNNs [25] or a Transformer decoder [24]. Next, we compute attention distribution $\left\{\alpha_{1, n}, \cdots, \alpha_{M, n}\right\}$ from the function that computes the attention distribution with $\boldsymbol{H}$ and $\boldsymbol{v}_{n}$. The attention distribution produces a weighted sum of the encoder states $\boldsymbol{d}_{n}$. Generation probabilities for the $n$-th token are produced with $\boldsymbol{d}_{n}$ and $\boldsymbol{v}_{n}$ by

$$
\begin{aligned}
P\left(y_{n} \mid y_{1: n-1}, \boldsymbol{X} ; \boldsymbol{\Theta}\right) & \\
= & P_{\text {gen }} G\left(y_{n}\right)+\left(1-P_{\text {gen }}\right) \sum_{m: x_{m}=y_{n}} \alpha_{m, n} \\
G\left(y_{n}\right)= & \operatorname{softmax}\left(\tanh \left(\left[\boldsymbol{d}_{n}^{\mathrm{T}}, \boldsymbol{v}_{n}^{\mathrm{T}}\right]^{\mathrm{T}} ; \theta_{\mathrm{dec}}\right) ; \theta_{\mathrm{dec}}\right), \\
P_{\text {gen }}= & \operatorname{sigmoid}\left(\tanh \left(\left[\boldsymbol{d}_{n}^{\mathrm{T}}, \boldsymbol{v}_{n}{ }^{\mathrm{T}}\right]^{\mathrm{T}} ; \theta_{\mathrm{dec}}\right) ; \theta_{\mathrm{dec}}\right),
\end{aligned}
$$

where $\tanh (\cdot), \operatorname{softmax}(\cdot)$ and $\operatorname{sigmoid}(\cdot)$ are linear transformational functions with a tanh, softmax and sigmoid activation. The copy mechanism enables switching probability $P_{\text {gen }}$ to choose whether to copy or generate tokens. Thus, $P_{\text {gen }}$ computes the weighted sum with generator distribution $G\left(y_{n}\right)$ and attention distribution and produces the prediction probability of the $n$-th token.

The model parameter set can be optimized from paired data $\mathcal{D}_{\mathrm{p}}$ $=\left\{\left(\boldsymbol{X}^{1}, \boldsymbol{Y}^{1}\right), \cdots,\left(\boldsymbol{X}^{\left|\mathcal{D}_{\mathrm{p}}\right|}, \boldsymbol{Y}^{\left|\mathcal{D}_{\mathrm{p}}\right|}\right)\right\}$. A loss function to optimize the model parameter set is defined as

$$
\mathcal{L}=\frac{1}{\left|\mathcal{D}_{\mathrm{p}}\right|} \sum_{d=1}^{\left|\mathcal{D}_{\mathrm{p}}\right|} \sum_{n=1}^{N} \log P\left(y_{n}^{d} \mid y_{1: n-1}^{d}, \boldsymbol{X}^{d} ; \boldsymbol{\Theta}\right) .
$$

\section{SELF-SUPERVISED LEARNING FOR POINTER-GENERATOR NETWORKS}

This section details self-supervised learning for pointer-generator networks. The denoising auto-encoder task is widely used for seq2seq self-supervised learning. In this task, the seq2seq model learns to reconstruct the original text given the corrupted original

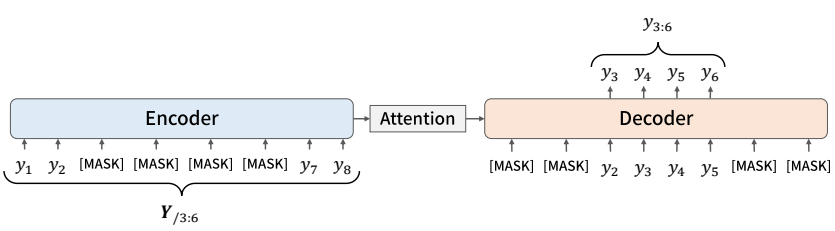

Fig. 2: Token span masking method.

text by using unpaired data. The seq 2 seq model can be fine-tuned with paired data for each subsequent task. In this paper, we use token span masking which is common with MASS [20] to corrupt the original text for the basic self-supervised learning strategy. We propose a self-supervised learning method for pointer-generator networks by devising a masking method for the token span masking.

\subsection{Basic self-supervised learning strategy}

We use token span masking in MASS [20] as a basic self-supervised learning strategy. In this method, given unpaired sentence $\boldsymbol{Y}$, we get $\boldsymbol{Y}_{/ a: b}$ where its fragment from position $a$ to $b$ are masked. Here, $0<a<b<n$ and $n$ is the number of tokens of sentence $\boldsymbol{Y}$. The number of tokens that are masked from position $a$ to $b$ is $k=b-a+$ 1 , and the length $k$ is roughly $50 \%$ of $n$. Position $a$ is selected from between the first token and the $n-k+1$-th token. $y_{a: b}$ indicates the sentence fragment of $\boldsymbol{Y}$ from $a$ to $b$. In addition, each of the selected masked token is either replaced with a [MASK] token, a random token or left unchanged. We describe details of this replacing method for the masked token in section 3.2. The seq2seq model is pre-trained by predicting the sentence fragment $y_{a: b}$ taking the masked sequence $\boldsymbol{Y}_{/ a: b}$ as input, as shown in Fig. 2 The model parameter set can be optimized from unpaired data $\mathcal{D}_{\mathrm{u}}=\left\{\boldsymbol{Y}^{1}, \cdots, \boldsymbol{Y}^{\left|\mathcal{D}_{\mathrm{u}}\right|}\right\}$. A loss function to pre-train the model parameter set is defined as

$$
\begin{aligned}
\mathcal{L}=\frac{1}{\left|\mathcal{D}_{\mathrm{u}}\right|} \sum_{d=1}^{\left|\mathcal{D}_{\mathrm{u}}\right|} \log P\left(y_{a: b}^{d} \mid y_{a-1}^{d}, \boldsymbol{Y}_{/ a: b}^{d} ; \boldsymbol{\Theta}\right) \\
\quad=\frac{1}{\left|\mathcal{D}_{\mathrm{u}}\right|} \sum_{d=1}^{\left|\mathcal{D}_{\mathrm{u}}\right|} \sum_{t=a}^{b} \log P\left(y_{t}^{d} \mid y_{a-1: t-1}^{d}, \boldsymbol{Y}_{/ a: b}^{d} ; \boldsymbol{\Theta}\right) .
\end{aligned}
$$

In this self-supervised learning, the encoder is encouraged to understand the meaning of unmasked tokens. Furthermore, the decoder is encouraged to extract more useful information from the encoder side by masking the decoder input tokens which are not masked in the encoder. If the decoder input tokens are not masked at all, it is assumed that the decoder uses abundant information from the preceding tokens rather than information from the encoder side. In addition, the decoder can learn more effective language modeling by predicting consecutive tokens in the decoder side rather than predicting discrete tokens.

\subsection{Masking methods}

We propose a self-supervised learning method for pointer-generator networks. In token span masking [20], each of the selected masked token is either replaced with a [MASK] token, a random token or left unchanged. We vary the percentage of these tokens and the replacing method to develop a suitable masking method for pointer-generator networks. This section describes the conventional method, MASS, two methods that varies the percentage of these replaced tokens in MASS and our proposed method, MAPGN. Table 1 summarizes each masking method.

MASS: In MASS, $80 \%$ of the masked tokens in the encoder are replaced by [MASK] tokens, $10 \%$ are replaced by random tokens, and $10 \%$ are unchanged. The random tokens are introduced on behalf 
Table 1: Summary of masking methods.

\begin{tabular}{|c|cccc|}
\hline & {$[$ MASK $]$} & Random & Unchanged & $\begin{array}{l}\text { Select random } \\
\text { tokens from }\end{array}$ \\
\hline \hline MASS-1 & $80 \%$ & $10 \%$ & $10 \%$ & all tokens \\
MASS-2 & $40 \%$ & $40 \%$ & $20 \%$ & all tokens \\
MASS-3 & $40 \%$ & - & $60 \%$ & - \\
\hline MAPGN & $40 \%$ & $40 \%$ & $20 \%$ & masking span \\
\hline
\end{tabular}

of the [MASK] token, considering that the [MASK] token does not appear during fine-tuning. These tokens are randomly selected from the vocabulary. In this paper, this masking method is referred to as MASS-1. Moreover, we prepare two methods that varies the percentage of these replaced tokens: MASS-2 and MASS-3. In MASS-2, $40 \%$ of the masked tokens in the encoder are replaced by [MASK] tokens, $40 \%$ are replaced by random tokens, and $20 \%$ are unchanged. In MASS-3, $40 \%$ of the masked tokens in the encoder are replaced by [MASK] tokens and $60 \%$ are unchanged.

MAPGN: Our MAsked Pointer-Generator Network (MAPGN) is an extension of MASS. In MAPGN, $40 \%$ of the masked tokens in the encoder are replaced by [MASK] tokens, $40 \%$ are replaced by random tokens, and $20 \%$ are unchanged. Key advance of MAPGN is that the random tokens are not selected from all tokens but from tokens in the masking span. For example, in Fig. 2, the random tokens are randomly selected from $\left\{y_{3}, y_{4}, y_{5}, y_{6}\right\}$. A reason why we use this masking method is detailed in section 3.3.

\subsection{Key idea}

We presume that MASS-1 is not suitable for pointer-generator network pre-training because the percentage of unchanged tokens in the masking span of the encoder is small and the model cannot learn to copy tokens explicitly. We assume that pointer-generator networks can learn to copy appropriate tokens from the input by increasing the percentage of unchanged tokens. Thus, it is assumed that MASS- 3 is suitable for pointer-generator network. However, if only unchanged tokens are increased, there is a possibility that the copy mechanism will be overfitting. Thus, MAPGN utilizes random tokens to learn to choose to copy or generate tokens effectively. By comparing MASS3 with MAPGN, we can verify that increasing unchanged tokens is not enough to pre-train the copy mechanism. In addition, MAPGN selects random tokens from masking span. In other words, although random tokens include tokens in the output sequence, these tokens do not have information on appropriate positions. Thus, these tokens are used to aid for copying tokens and conceal the position of tokens that should be copied. The role of the random tokens in MAPGN is not only to mask the token but also encourage to determine whether to copy. By comparing MASS-2 with MAPGN, we can validate the selecting of random tokens from the masking span.

\section{EXPERIMENTS}

This section describes the experimental details of pre-training and fine-tuning on spoken-text normalization tasks. In particular, we chose dialect conversion and spoken-to-written style conversion tasks in Japanese. In the dialect conversion task, Japanese dialect is converted into standard Japanese. In the spoken-to-written style conversion task, spoken-style text produced by an automatic speech recognition system is converted into written-style text with correct punctuations and no disfluencies.

\subsection{Datasets}

Pre-training: We prepared a large-scale Japanese web text as the unpaired written-style text data. The web text was downloaded from various topic web pages using our home-made crawler. The downloaded pages were filtered in such a way that HTML tags, Javascript codes and other parts that were not useful for these tasks were excluded. Finally, we prepare one million sentences for pre-training.

Fine-tuning on dialect conversion task: We prepared paired data of a Japanese dialect (Tohoku-ben) and standard Japanese using crowd-sourcing. We divided the data into a training set, validation set, and test set. The training set contained 4,492 sentences and we divided the training set into 1,000 and 4,000 sentences to investigate the difference of performance with different amount of training data. The validation set have 1,702 sentences and the test set have 700 sentences.

Fine-tuning on spoken-to-written style conversion task: We used the parallel corpus for Japanese spoken-to-written style conversion (CJSW) [26]. Although the CJSW has four domains, we only used the data from one domain (call center dialogue) for training to compare the in-domain (ID) task and the out-of-domain (OOD) task. The training set has 8,169 sentences, which we divided into increments of 1,000 between 1,000 and 8,000 sentences for the same reason as the dialect conversion task. A validation set of call center dialogue containing 584 sentences was used for training. We used all domain test sets: the call center dialogue test set for the ID task and all other test sets for the OOD task. The test sets were divided in accordance with |26|. The datasets were paired data of spoken-style text (manual transcriptions of speech) and written-style text (created by crowd-sourcing).

\subsection{Setups}

We pre-trained attention-based encoder-decoder networks [27] and pointer-generator networks [13] with four pre-training methods. We used the following configurations. We used pre-trained 512 dimensional word embeddings using continuous bag-of-words [28]. In the encoder, a 4-layer bidirectional long short-term memory RNN (LSTM-RNN) with 256 units was introduced. In the decoder, a 2-layer unidirectional LSTM-RNN with 256 units was introduced. We used an additive attention mechanism [29]. The output unit size (which corresponded to the number of tokens in the training set of word embeddings) was set to 5,640. To train these networks, we used the adam optimizer and label smoothing with a smoothing parameter of 0.1 . We set the mini-batch size to 64 sentences and the dropout rate in each LSTM-RNN to 0.1. For the mini-batch training, we truncated each sentence to 200 tokens. All trainable parameters were randomly initialized. We used characters as tokens and these pre-trained networks for two tasks in common.

In fine-tuning, we used the attention-based encoder-decoder network and the pointer-generator network which are transferred pretrained model parameter sets. We constructed these two networks without pre-training as the baseline. These model configurations were the same as that of the pre-training model, and all trainable parameters were randomly initialized. In the evaluation, we calculated automatic evaluation scores in three metrics: BLEU-3 [30], ROUGE-L [31], and METEOR [32].

\subsection{Results}

Table 2 and Table 3 show the experimental results of the dialect conversion task and the spoken-to-written style conversion task, respectively. Fig. 3 shows the BLEU-3 score with all training data in spoken-to-written style conversion task. Table 2 shows that MAPGN for pointer-generator networks outperformed other masking methods in all evaluation metrics. Although MASS-3 was the best performance in encoder-decoder networks, it was the least performance in pointer-generator networks. The results were the same whether 1,000 or 4,000 sentences were used as training; however, the performance of MAPGN improved when 1,000 sentences were used. 
Table 2: Results of dialect conversion task with 1,000 and 4,000 sentences of training data.

\begin{tabular}{|c|c|c|c|c|c|c|c|}
\hline & \multicolumn{4}{|c|}{1,000 sentences } & \multicolumn{3}{|c|}{4,000 sentences } \\
\hline Masking method & & BELU-3 & ROUGE-L & METEOR & BELU-3 & ROUGE-L & METEOR \\
\hline \multirow{2}{*}{ baseline } & 1). & 0.112 & 0.350 & 0.241 & 0.441 & 0.618 & 0.523 \\
\hline & 2). & 0.332 & 0.543 & 0.411 & 0.599 & 0.749 & 0.708 \\
\hline \multirow{2}{*}{ MASS-1 } & 1). & 0.447 & 0.635 & 0.550 & 0.614 & 0.755 & 0.719 \\
\hline & 2). & 0.494 & 0.672 & 0.598 & 0.650 & 0.785 & 0.766 \\
\hline \multirow{2}{*}{ MASS-2 } & 1). & 0.476 & 0.657 & 0.573 & 0.610 & 0.753 & 0.714 \\
\hline & 2). & 0.497 & 0.674 & 0.601 & 0.647 & 0.783 & 0.728 \\
\hline \multirow{2}{*}{ MASS-3 } & 1). & 0.502 & 0.677 & 0.597 & 0.639 & 0.776 & 0.756 \\
\hline & 2). & 0.454 & 0.646 & 0.564 & 0.622 & 0.763 & 0.728 \\
\hline \multirow{2}{*}{ MAPGN } & 1). & 0.462 & 0.652 & 0.571 & 0.613 & 0.761 & 0.731 \\
\hline & 2). & 0.535 & 0.704 & 0.646 & 0.656 & 0.789 & 0.770 \\
\hline
\end{tabular}

1). Attention-based encoder-decoder network 2). Pointer-generator network

Table 3: Results of spoken-to-written style conversion task with 1,000 and 8,000 sentences of training data.

\begin{tabular}{|c|c|c|c|c|c|c|c|c|c|c|c|c|c|}
\hline \multirow{3}{*}{ Masking method } & \multicolumn{7}{|c|}{1,000 sentences } & \multicolumn{6}{|c|}{8,000 sentences } \\
\hline & & \multicolumn{2}{|c|}{ BELU-3 } & \multicolumn{2}{|c|}{ ROUGE-L } & \multicolumn{2}{|c|}{ METEOR } & \multicolumn{2}{|c|}{ BELU-3 } & \multicolumn{2}{|c|}{ ROUGE-L } & \multicolumn{2}{|c|}{$\overline{\text { METEOR }}$} \\
\hline & & ID & OOD & ID & OOD & ID & OOD & ID & OOD & ID & OOD & ID & OOD \\
\hline \multirow{2}{*}{ baseline } & 1). & 0.177 & 0.061 & 0.360 & 0.247 & 0.296 & 0.235 & 0.651 & 0.245 & $\overline{0.736}$ & 0.412 & 0.809 & 0.394 \\
\hline & 2). & 0.493 & 0.278 & 0.651 & 0.474 & 0.676 & 0.490 & 0.683 & 0.474 & 0.758 & 0.599 & 0.839 & 0.671 \\
\hline \multirow{2}{*}{ MASS-1 } & 1). & 0.515 & 0.189 & 0.640 & 0.371 & 0.674 & 0.365 & 0.700 & 0.355 & 0.770 & 0.495 & 0.858 & 0.515 \\
\hline & 2). & 0.555 & 0.259 & 0.675 & 0.449 & 0.718 & 0.473 & 0.700 & 0.533 & 0.772 & 0.633 & 0.860 & 0.696 \\
\hline \multirow{2}{*}{ MASS-2 } & 1). & 0.519 & 0.182 & 0.645 & 0.370 & 0.681 & 0.372 & 0.705 & 0.387 & 0.772 & 0.517 & 0.867 & 0.540 \\
\hline & 2). & 0.523 & 0.224 & 0.651 & 0.421 & 0.692 & 0.449 & 0.707 & 0.537 & 0.771 & 0.638 & 0.864 & 0.706 \\
\hline \multirow{2}{*}{ MASS-3 } & 1). & 0.571 & 0.242 & 0.682 & 0.419 & 0.728 & 0.416 & 0.703 & 0.429 & 0.771 & 0.545 & 0.865 & 0.577 \\
\hline & 2). & 0.439 & 0.138 & 0.592 & 0.351 & 0.592 & 0.347 & 0.698 & 0.467 & 0.767 & 0.591 & 0.855 & 0.639 \\
\hline \multirow{2}{*}{ MAPGN } & 1). & 0.522 & 0.197 & 0.659 & 0.400 & 0.703 & 0.420 & 0.696 & 0.386 & 0.768 & 0.522 & 0.859 & 0.539 \\
\hline & 2). & 0.583 & 0.342 & 0.696 & 0.509 & 0.752 & 0.542 & 0.711 & 0.542 & 0.779 & 0.648 & 0.872 & 0.713 \\
\hline
\end{tabular}

$\begin{array}{ll}\text { 1). Attention-based encoder-decoder network } & \text { 2). Pointer-generator network }\end{array}$

Table 3 shows that MAPGN for pointer-generator networks outperformed other masking methods in all metrics. Fig. 3 shows that in encoder-decoder networks, all masking methods improved the baseline performance significantly. Moreover, MASS-3 was the best performance for any amount of training data. In pointer-generator networks, MAPGN yield the best performance and the performance was improved more significantly in the OOD task than in the ID task. In both ID and OOD tasks, the performance difference between each masking method decreased as the amount of paired training data increased.

The results of the two spoken-text normalization tasks largely followed the same trend. The results of encoder-decoder networks show that MASS-3 outperformed all other methods. In other words, the pre-training method which learned to actively output the same tokens in the input sequence was more effective. It is assumed that the method to actively copy tokens is effective for spoken-text normalization tasks using encoder-decoder networks. Next, a notable point of pre-training in pointer-generator networks results is that each masking method performed differently. This indicates that the masking method is important for pointer-generator network pre-training. For example, MASS-3 was the least performance and MASS-2 was less effective than MAPGN. We can infer that even if the pointer-generator network simply pre-trains to copy tokens actively, the copy mechanism learns to copy tokens that do not need to be copied. Moreover, we assume that the networks can be pre-trained to copy or not effectively by selecting random tokens from tokens in the masking span rather than from all tokens in the model. Finally, the results of the pointer-generator networks show that MAPGN outperformed other masking methods. However, in the ID task, since pointer-generator networks are suitable for spokenstyle normalization task and the baseline performance is improved as the amount of paired training data increases, the effectiveness of pre-training for pointer-generator networks decreases. On the other hand, in the OOD task, pre-training is effective significantly even if the amount of paired training data increases. Thus, MAPGN is an effective pre-training method for pointer-generator networks if the amount of paired training data is small or in OOD tasks.
Attention-based encoder-decoder networks Pointer-generator networks
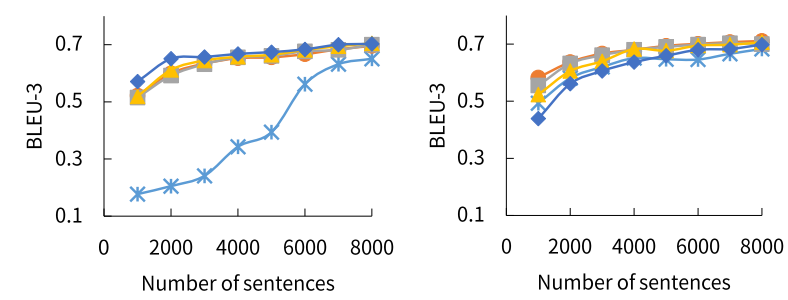

Out-of-domain task

Attention-based encoder-decoder networks

Pointer-generator networks
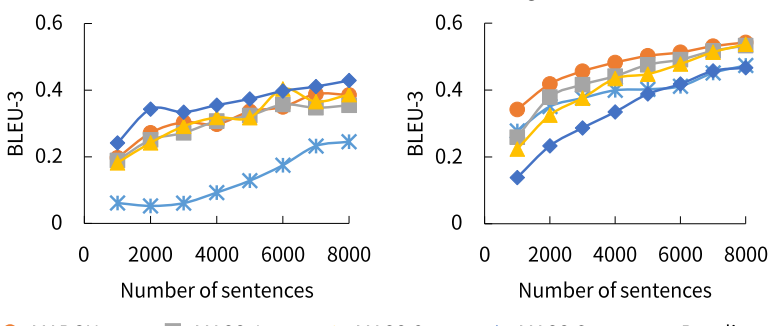

- MAPGN - MASS-1 $\neq$ MASS-2 $\rightarrow$ MASS-3 * Baseline

Fig. 3: BLEU-3 score of spoken-to-written style conversion task.

\section{CONCLUSION}

This paper proposed MAsked Pointer-Generator Network (MAPGN), a self-supervised learning method for pointer-generator networks. While conventional self-supervised learning methods do not support to explicitly train a copy mechanism in the pointer-generator networks, the proposed method can train the copy mechanism efficiently by learning to choose whether to copy or generate tokens against masking span. Experiments demonstrated that MAPGN outperformed the conventional methods in two spoken-text normalization tasks and was especially effective if the amount of paired training data is small and in OOD tasks. We concluded that MAPGN is suitable for pre-training pointer-generator networks and effective when paired data set is limited. 


\section{REFERENCES}

[1] Bo Li, Tara Sainath, Arun Narayanan, Joe Caroselli, Michiel Bacchiani, Ananya Misra, Izhak Shafran, Hasim Sak, Golan Pundak, Kean Chin, Khe Chai Sim, Ron J. Weiss, Kevin Wilson, Ehsan Variani, Chanwoo Kim, Olivier Siohan, Mitchel Weintraub, Erik McDermott, Rick Rose, and Matt Shannon, "Acoustic modeling for google home," in Proc. Annual Conference of the International Speech Communication Association (INTERSPEECH), 2017.

[2] Amanda Purington, Jessie Taft, Shruti Sannon, Natalya (Natalie) Bazarova, and Samuel Taylor, "'alexa is my new bff": Social roles, user satisfaction, and personification of the amazon echo," in Proc. Conference Extended Abstracts on Human Factors in Computing Systems (CHI), 2017, pp. 2853-2859.

[3] Guokan Shang, Wensi Ding, Zekun Zhang, Antoine Jean-Pierre Tixier, Polykarpos Meladianos, Michalis Vazirgiannis, and Jean-Pierre Lorré, "Unsupervised abstractive meeting summarization with multi-sentence compression and budgeted submodular maximization," in Proc. Annual Meeting of the Association for Computational Linguistics (ACL), 2018.

[4] Manling Li, Lingyu Zhang, Heng Ji, and Richard J Radke, "Keep meeting summaries on topic: Abstractive multi-modal meeting summarization," in Proc. Annual Meeting of the Association for Computational Linguistics (ACL), 2019, pp. 2190-2196.

[5] Sander Wubben, Antal Van Den Bosch, and Emiel Krahmer, "Paraphrase generation as monolingual translation: Data and evaluation," in Proc. International Natural Language Generation Conference (INLG), 2010, pp. 203-207.

[6] Ilya Sutskever, Oriol Vinyals, and Quoc V Le, "Sequence to sequence learning with neural networks," in Proc. International Conference on Neural Information Processing Systems (NIPS), 2014, pp. 3104-3112.

[7] Romain Paulus, Caiming Xiong, and Richard Socher, "A deep reinforced model for abstractive summarization," in Proc. International Conference on Learning Representations (ICLR), 2017.

[8] Yen-Chun Chen and Mohit Bansal, "Fast abstractive summarization with reinforce-selected sentence rewriting," in Proc. Association for Computational Linguistics (ACL), 2018, pp. 675-686.

[9] Aaditya Prakash, Sadid A Hasan, Kathy Lee, Vivek Datla, Ashequl Qadir, Joey Liu, and Oladimeji Farri, "Neural paraphrase generation with stacked residual lstm networks," in Proc. International Conference on Computational Linguistics (COLING), 2016, pp. 2923-2934.

[10] Shuming Ma, Xu Sun, Wei Li, Sujian Li, Wenjie Li, and Xuancheng Ren, "Query and output: Generating words by querying distributed word representations for paraphrase generation," in Proc. Conference of the North American Chapter of the Association for Computational Linguistics: Human Language Technologies (NAACL-HLT), 2018, pp. 196-206.

[11] Shaolei Wang, Wanxiang Che, and Ting Liu, "A neural attention model for disfluency detection," in Proc. International Conference on Computational Linguistics (COLING), 2016, pp. 278-287.

[12] Qianqian Dong, Feng Wang, Zhen Yang, Wei Chen, Shuang Xu, and $\mathrm{Bo} \mathrm{Xu}$, "Adapting translation models for transcript disfluency detection," in Proc. Thirty-Third AAAI Conference on Artificial Intelligence (AAAI), 2019, pp. 6351-6358.

[13] Abigail See, Peter J Liu, and Christopher D Manning, "Get to the point: Summarization with pointer-generator networks," in Proc. Annual Meeting of the Association for Computational Linguistic (ACL), 2017, pp. 1073-1083.

[14] Zhengyuan Liu, Angela Ng, Sheldon Lee, Ai Ti Aw, and Nancy F Chen, "Topic-aware pointer-generator networks for summarizing spoken conversations," in Proc. IEEE Automatic Speech Recognition and Understanding Workshop (ASRU), 2019.

[15] Wenjun Zhao, Meina Song, and E. Haihong, "Summarization with highway condition radom pointer-generator network," in Proc. International Conference on Algorithms, Computing and Artificial Intelligence (ACAI), 2018, pp. 1-5.
[16] Mana Ihori, Akihiko Takashima, and Ryo Masumura, "Large-context pointer-generator networks for spoken-to-written style conversion," in Proc. International Conference on Acoustics, Speech and Signal Processing (ICASSP), 2020, pp. 8189-8193.

[17] Jacob Devlin, Ming-Wei Chang, Kenton Lee, and Kristina Toutanova, "Bert: Pre-training of deep bidirectional transformers for language understanding," arXiv preprint arXiv:1810.04805 2018.

[18] Matthew Peters, Mark Neumann, Mohit Iyyer, Matt Gardner, Christopher Clark, Kenton Lee, and Luke Zettlemoyer, "Deep contextualized word representations," in Proceedings of the 2018 Conference of the North American Chapter of the Association for Computational Linguistics: Human Language Technologies, Volume 1 (Long Papers), 2018, pp. 2227-2237.

[19] Alec Radford, Jeffrey Wu, Rewon Child, David Luan, Dario Amodei, and Ilya Sutskever, "Language models are unsupervised multitask learners," OpenAI blog, p. 9, 2019.

[20] Kaitao Song, Xu Tan, Tao Qin, Jianfeng Lu, and Tie-Yan Liu, "Mass: Masked sequence to sequence pre-training for language generation," in Proc. International Conference on Machine Learning (ICML), 2019, pp. 5926-5936.

[21] Yinhan Liu, Jiatao Gu, Naman Goyal, Xian Li, Sergey Edunov, Marjan Ghazvininejad, Mike Lewis, and Luke Zettlemoyer, "Multilingual denoising pre-training for neural machine translation," arXiv preprint arXiv:2001.08210 2020.

[22] Liang Wang, Wei Zhao, Ruoyu Jia, Sujian Li, and Jingming Liu, "Denoising based sequence-to-sequence pre-training for text generation," in Proc. Conference on Empirical Methods in Natural Language Processing and the 9th International Joint Conference on Natural Language Processing (EMNLP-IJCNLP), 2019, pp. 3994-4006.

[23] Mike Schuster and Kuldip K Paliwal, "Bidirectional recurrent neural networks," IEEE transactions on Signal Processing, pp. 2673-2681, 1997.

[24] Ashish Vaswani, Noam Shazeer, Niki Parmar, Jakob Uszkoreit, Llion Jones, Aidan N Gomez, Łukasz Kaiser, and Illia Polosukhin, "Attention is all you need," in Proc. Advances in neural information processing systems (NIPS), 2017, pp. 5998-6008.

[25] Sepp Hochreiter and Jürgen Schmidhuber, "Long short-term memory," Neural computation, pp. 1735-1780, 1997.

[26] Mana Ihori, Akihiko Takashima, and Ryo Masumura, "Parallel corpus for Japanese spoken-to-written style conversion," in Proc. Language Resources and Evaluation Conference (LREC), 2020, pp. 6346-6353.

[27] Thang Luong, Hieu Pham, and Christopher D. Manning, "Effective approaches to attention-based neural machine translation," in Proc. Conference on Empirical Methods in Natural Language Processing (EMNLP), 2015, pp. 1412-1421.

[28] Tomas Mikolov, Kai Chen, Greg Corrado, and Jeffrey Dean, "Efficient estimation of word representations in vector space," in Proc. Workshop at International Conference on Learning Representations (ICLR), 2013.

[29] Dzmitry Bahdanau, Kyunghyun Cho, and Yoshua Bengio, "Neural machine translation by jointly learning to align and translate," in Proc. International Conference on Learning Representations (ICLR), 2015, pp. 1-15.

[30] Kishore Papineni, Salim Roukos, Todd Ward, and Wei-Jing Zhu, "Bleu: a method for automatic evaluation of machine translation," in Proc. Annual Meeting on Association for Computational Linguistics (ACL), 2002, pp. 311-318.

[31] Chin-Yew Lin and Franz Josef Och, "Automatic evaluation of machine translation quality using longest common subsequence and skip-bigram statistics," in Proc. Annual Meeting on Association for Computational Linguistics (ACL), 2004, pp. 605-612.

[32] Satanjeev Banerjee and Alon Lavie, "METEOR: An automatic metric for MT evaluation with improved correlation with human judgments," in Proc. the ACL Workshop on Intrinsic and Extrinsic Evaluation Measures for Machine Translation and/or Summarization, 2005, pp. 65-72. 